\title{
Wolbachia diversity and cytoplasmic incompatibility patterns in Culex pipiens populations in Turkey
}

\author{
Mine Altinli ${ }^{1 *}$ (D), Filiz Gunay ${ }^{2}$, Bulent Alten², Mylene Weill ${ }^{1}$ and Mathieu Sicard ${ }^{1 *}$
}

\begin{abstract}
Background: Wolbachia are maternally transmitted bacteria that can manipulate their hosts' reproduction causing cytoplasmic incompatibility (Cl). Cl is a sperm-egg incompatibility resulting in embryonic death. Due to this sterilising effect on mosquitoes, Wolbachia are considered for vector control strategies. Important vectors for arboviruses, filarial nematodes and avian malaria, mosquitoes of Culex pipiens complex are suitable for Wolbachia-based vector control. They are infected with Wolbachia wPip strains belonging to five genetically distinct groups (wPip-l to V) within the Wolbachia B supergroup. Cl properties of wPip strongly correlate with this genetic diversity: mosquitoes infected with wPip strains from a different wPip group are more likely to be incompatible with each other. Turkey is a critical spot for vector-borne diseases due to its unique geographical position as a natural bridge between Asia, Europe and Africa. However, general wPip diversity, distribution and Cl patterns in natural CX. pipiens (s.l.) populations in the region are unknown. In this study, we first identified wPip diversity in Turkish CX. pipiens (s.l.) populations, by assigning them to one of the five groups within wPip (wPip-Ito V). We further investigated Cl properties between different wPip strains from this region.
\end{abstract}

Results: We showed a wPip fixation in CX. pipiens (s.l.) populations in Turkey by analysing 753 samples from 59 sampling sites. Three wPip groups were detected in the region: WPip-I, wPip-II and WPip-IV. The most dominant group was wPip-II. While WPip-IV was restricted to only two locations, wPip-I and wPip-II had wider distributions. Individuals infected with WPip-II were found co-existing with individuals infected with WPip-I or WPip-IV in some sampling sites. Two mosquito isofemale lines harbouring either a wPip-I or a wPip-II strain were established from a population in northwestern Turkey. Reciprocal crosses between these lines showed that they were fully compatible with each other but bidirectionally incompatible with wPip-IV Istanbul infected line.

Conclusion: Our findings reveal a high diversity of wPip and Cl properties in CX. pipiens (s.l.) populations in Turkey. Knowledge on naturally occurring Cl patterns caused by wPip diversity in Turkey might be useful for Cx. pipiens (s.l.) control in the region.

Keywords: Wolbachia, Culex pipiens, Cytoplasmic incompatibility, Turkey, Vector control

\section{Background}

First discovered in Culex pipiens (s.l.) mosquitoes [1], the $\alpha$-proteobacterium Wolbachia pipientis is one of the most common vertically transmitted cytoplasmic symbionts. Indeed, meta-analysis predicts Wolbachia infection in up to $50 \%$ of the arthropod species [2]. The success of

\footnotetext{
* Correspondence: mine.altinli@umontpellier.fr;

mathieu.sicard@umontpellier.fr

${ }^{1}$ Institut des Sciences de l'Evolution de Montpellier (CNRS-Université de

Montpellier-IRD-EPHE), Montpellier, France

Full list of author information is available at the end of the article
}

their vertical transmission mainly relies on their host reproduction manipulation strategies; parthenogenesis induction, feminization, male killing and cytoplasmic incompatibility (CI) [3]. CI, the most common of these strategies, is modelled by a modification-rescue (mod-resc) system where Wolbachia modifies sperm of infected males (mod function), and only a compatible Wolbachia strain in the eggs can rescue (resc function) this modification [4]. Consequently, Wolbachia causes conditional sterility in crosses either between uninfected females and infected males [5] 
or like in the case of $C x$. pipiens (s.l.), between females and males infected with incompatible Wolbachia strains [6, 7].

Consisting of several species, including important disease vectors with worldwide distribution (e.g. Cx. pipiens and $C x$. quinquefasciatus) [8], mosquitoes of $C x$. pipiens complex have a unique relationship with their endosymbiont Wolbachia (wPip). wPip is fixed in natural Cx. pipiens (s.l.) populations where they induce the most complex CI relationships yet described among arthropods, including uni and bidirectional incompatibility [7, 9-12]. So far, reciprocal crosses between many isofemale lines and four reference lines showed eight different $\bmod$ and four different resc functions in Cx. pipiens (s.l.), resulting in the definition of 14 different cytotypes throughout the world [7].

In contrast to this observed diversity of $\mathrm{CI}$ patterns, $C x$. pipiens $w$ Pip strains are closely related, and all belong to a clade within Wolbachia B supergroup [12-14]. However, recent studies of fast evolving markers showed the presence of many genetically distinct $w$ Pip strains in $C x$. pipiens (s.l.) mosquitoes [10,12, 15] distributed in five distinct phylogenetic groups (wPip-I to V) [12]. Using a PCR/RFLP assay based on pk1 gene, encoding proteins with ankyrin motifs, a $w$ Pip strain can be assigned to one of these five groups $[12,16]$. A study of the $w$ Pip worldwide distribution showed an important spatial structure of $w$ Pip groups [16]. For instance, only $w$ Pip-I was found in sub-Saharan Africa, South America and Southeast Asia, while $w$ Pip-III was mainly observed in North America. Strains belonging to the $w$ Pip-II group were mostly found in western Europe and $w$ Pip-V in Asia. $w$ Pip-IV group strains exhibit a patchy distribution in Europe, North Africa and Asia [16]. Also, Wolbachia genetic diversity and their CI patterns strongly correlate; most $w$ Pip strains from the same group render their host compatible with each other (except few unidirectional incompatibilities) whereas those from different groups often lead to unidirectional or bidirectional incompatibilities [7]. Recently this huge diversity of $\mathrm{CI}$ patterns observed in Cx. pipiens has been explained by the amplification and the diversity of an operon in $w$ Pip strains' genomes [17] composed of cidA and $\operatorname{cidB}$ genes involved in Wolbachia induced CI $[18,19]$. No effect of host genetic background on the CI patterns [20] and no multiple infections by several strains have ever been shown $[12,16,21]$.

Being a natural bridge between Africa, Asia and Europe, Turkey is a critical spot for many emerging and reemerging vector-borne diseases $[22,23]$ and for the diversity of the vectors that transmit these diseases [23]. For instance, high diversity and abundance of $C x$. pipiens (s.l.) species have been recorded in the area including $C x$. quinquefasciatus, $C x$. pipiens and its physiological variant $C x$. pipiens f. molestus [24]. Arboviruses such as West Nile virus, mainly transmitted by these mosquitoes, have also been shown to circulate in Turkey [25-28]. Therefore, understanding $w$ Pip diversity and their CI properties of $w$ Pip to control Cx. pipiens (s.l.) populations in Turkey is a cornerstone for vector control in the region and prevention of putative epidemics extending through Europe, Asia and northern Africa. This knowledge can contribute to the biological vector control techniques using CI properties such as incompatible insect technique (IIT). IIT, the mass release of males harbouring incompatible Wolbachia into focal populations, has been shown to successfully decrease the female reproduction by sterilisation and reduce the pest/vector populations [29-34]. Nevertheless, our knowledge of the $w$ Pip genetic diversity and the CI patterns in Turkey is yet limited to only one line established with samples collected in Istanbul in 2003 [35].

Here, we collected and analysed 753 Cx. pipiens (s.l.) individuals (larvae and adults) from natural populations across Turkey. We studied (i) the $w$ Pip diversity in this geographically critical region in the crossroads of three continents, (ii) the $\mathrm{CI}$ relationships between $\mathrm{Cx}$. pipiens lines from Turkey and (iii) the CI relationships between Turkish lines and reference lines to compare their $\mathrm{CI}$ properties to previously characterized mod (male crossing type) and resc (female crossing type) functions. Taken together, these results might be used in integrated vector control programs against Cx. pipiens (s.l.) in Turkey.

\section{Methods}

\section{Sample collection and identification}

A total of 753 samples from 59 different sampling sites in Turkey were tested for Wolbachia diversity. Most of these samples $(n=677)$ were collected during the larval stage, between July to September 2016 (Table 1). The rest of the samples has been collected as adults, using adult light traps, from May to September (2012-2015) (Table 1, Fig. 1). All of the sampling sites were situated outdoors with the only exception of sample site 16 (Table 1). Collected larvae and adults were morphologically identified as Culex pipiens (s.l.) / Cx. torrentium [36]. As the samples were only morphologically identified, we used $C x$. pipiens (s.l.) to refer to $C x$ pipiens assemblage that includes both $C x$. pipiens and $C x$. quinquefasciatus, their hybrids and physiological forms $[21,35]$. Samples were stored in $70 \%$ ethanol until DNA extraction before testing them for the $w$ Pip presence and genetic characterisation.

\section{Isofemale lines}

For analysing the CI patterns induced by the $w$ Pip strains belonging to different groups found in Turkey, egg rafts and larvae were collected from a population in Thrace region of Turkey, in Tekirdag (Table 1 sampling site 52, Fig. 1). Collected larvae were reared to adults in insectary conditions (at $25 \pm 2{ }^{\circ} \mathrm{C}$ and $75 \pm 2 \%$ relative 
Table 1 Sampling sites, year, life stage and wPip groups of Culex pipiens (s.l.) individuals collected from Turkey

\begin{tabular}{|c|c|c|c|c|c|c|c|c|c|}
\hline Province & Sampling site & Latitude $\left({ }^{\circ} \mathrm{N}\right)$ & Longitude ( $\left.{ }^{\circ} \mathrm{E}\right)$ & Breeding site type & Stage & Year & wPip-I & wPip-II & WPip-IV \\
\hline Adana & 1 & 36.9475 & 35.485 & Rural & $A$ & 2013 & - & 5 & - \\
\hline \multirow[t]{2}{*}{ Ankara } & 2 & 39.8730 & 32.7370 & Suburban & $\mathrm{L}$ & 2016 & - & 27 & - \\
\hline & 3 & 39.8716 & 32.7356 & Suburban & A & 2014 & 2 & 3 & - \\
\hline \multirow[t]{12}{*}{ Artvin } & 4 & 41.3884 & 41.4335 & Suburban & L & 2016 & 1 & 35 & - \\
\hline & 5 & 41.4919 & 41.5367 & Suburban & L & 2016 & - & 5 & - \\
\hline & 6 & 41.3833 & 41.5716 & Rural & $L$ & 2016 & - & 5 & - \\
\hline & 7 & 41.3651 & 41.6835 & Suburban & $L$ & 2016 & 1 & 20 & - \\
\hline & 8 & 41.3911 & 41.6933 & Rural & L & 2016 & 7 & 17 & - \\
\hline & 9 & 41.3646 & 41.6686 & Suburban & L & 2016 & - & 3 & - \\
\hline & 10 & 41.3742 & 41.6235 & Rural & L & 2016 & 21 & 4 & - \\
\hline & 11 & 41.3192 & 41.3534 & Rural & L & 2016 & 12 & - & - \\
\hline & 12 & 41.3178 & 41.3412 & Rural & L & 2016 & 10 & - & - \\
\hline & 13 & 41.3274 & 41.3022 & Rural & L & 2016 & 36 & - & - \\
\hline & 14 & 40.7823 & 41.4991 & Rural & A & 2013 & - & 1 & - \\
\hline & 15 & 41.3928 & 41.6937 & Rural & A & 2013 & 4 & 1 & - \\
\hline Aydin & 16 & 37.4123 & 27.3612 & Rural & A & 2012 & 3 & - & - \\
\hline \multirow[t]{2}{*}{ Bartin } & 17 & 41.8383 & 32.7115 & Rural & L & 2016 & 10 & - & - \\
\hline & 18 & 41.7411 & 32.3827 & Suburban & L & 2016 & 5 & - & - \\
\hline Bursa & 19 & 40.0948 & 29.4912 & Urban & A & 2013 & 1 & 9 & - \\
\hline Duzce & 20 & 41.0708 & 30.9645 & Rural & L & 2016 & 7 & 3 & - \\
\hline \multirow[t]{5}{*}{ Edirne } & 21 & 41.6134 & 26.9656 & Rural & L & 2016 & - & 16 & 11 \\
\hline & 22 & 41.6731 & 26.9809 & Rural & $L$ & 2016 & 11 & - & - \\
\hline & 23 & 41.6635 & 26.5078 & Suburban & L & 2016 & - & 17 & - \\
\hline & 24 & 40.8548 & 26.6897 & Suburban & A & 2012 & - & 4 & - \\
\hline & 25 & 40.9404 & 26.4382 & Rural & A & 2012 & - & 1 & - \\
\hline Erzincan & 26 & 39.2476 & 38.5050 & Rural & A & 2014 & - & 3 & - \\
\hline \multirow[t]{3}{*}{ Eskisehir } & 27 & 39.7950 & 30.4972 & Urban & $L$ & 2016 & - & 27 & - \\
\hline & 28 & 39.2051 & 30.7145 & Rural & A & 2013 & - & 2 & - \\
\hline & 29 & 39.7098 & 30.4035 & Rural & L & 2016 & - & 5 & - \\
\hline Hatay & 30 & 36.2516 & 36.3166 & Suburban & $A$ & 2015 & 1 & - & - \\
\hline \multirow[t]{4}{*}{ Istanbul } & 31 & 40.9481 & 29.3050 & Urban & L & 2016 & - & 38 & - \\
\hline & 32 & 40.9418 & 29.3016 & Urban & L & 2016 & - & 44 & - \\
\hline & 33 & 40.9796 & 29.0557 & Urban & L & 2016 & 8 & 24 & - \\
\hline & 34 & 41.0783 & 29.0136 & Urban & A & 2016 & - & - & 1 \\
\hline Kahramanmaras & 35 & 37.5588 & 36.9737 & Urban & A & 2015 & 3 & - & - \\
\hline Karadeniz Ereglisi & 36 & 41.2824 & 31.4241 & Urban & $L$ & 2016 & 6 & - & - \\
\hline Kastamonu & 37 & 41.8886 & 32.9995 & Suburban & L & 2016 & 5 & - & - \\
\hline \multirow[t]{3}{*}{ Kirklareli } & 38 & 41.8458 & 27.8065 & Rural & L & 2016 & - & 10 & - \\
\hline & 39 & 41.5239 & 27.0258 & Rural & A & 2015 & - & 6 & - \\
\hline & 40 & 41.8300 & 27.0638 & Rural & A & 2015 & - & 2 & - \\
\hline Kocaeli & 41 & 40.6882 & 30.2797 & Urban & L & 2016 & - & 15 & - \\
\hline Malatya & 42 & 38.8180 & 37.9769 & Rural & A & 2014 & - & 1 & - \\
\hline \multirow[t]{2}{*}{ Mardin } & 43 & 37.5607 & 40.8865 & Rural & A & 2013 & 5 & - & - \\
\hline & 44 & 37.5477 & 40.9588 & Rural & $A$ & 2013 & 5 & - & - \\
\hline
\end{tabular}


Table 1 Sampling sites, year, life stage and wPip groups of Culex pipiens (s.l.) individuals collected from Turkey (Continued)

\begin{tabular}{|c|c|c|c|c|c|c|c|c|c|}
\hline Province & Sampling site & Latitude $\left({ }^{\circ} \mathrm{N}\right)$ & Longitude $\left({ }^{\circ} \mathrm{E}\right)$ & Breeding site type & Stage & Year & wPip-I & wPip-II & wPip-IV \\
\hline Osmaniye & 45 & 37.1375 & 36.2010 & Suburban & $A$ & 2015 & 1 & 1 & - \\
\hline Sakarya & 46 & 41.0719 & 30.8454 & Suburban & L & 2016 & - & 5 & - \\
\hline Samsun & 47 & 41.3689 & 36.2289 & Urban & A & 2013 & 3 & - & - \\
\hline \multirow[t]{2}{*}{ Sinop } & 48 & 41.9915 & 35.0908 & Suburban & L & 2016 & 1 & 5 & - \\
\hline & 49 & 41.9309 & 34.5819 & Suburban & L & 2016 & 6 & - & - \\
\hline \multirow[t]{3}{*}{ Tekirdag } & 50 & 41.1503 & 27.8522 & Urban & A & 2012 & - & 3 & - \\
\hline & 51 & 40.8886 & 27.4604 & Urban & A & 2015 & - & 6 & - \\
\hline & $52^{\mathrm{a}}$ & 41.0259 & 27.5805 & Urban & L & 2016 & na & na & na \\
\hline Tokat & 53 & 40.1916 & 35.5139 & Rural & A & 2014 & - & 1 & - \\
\hline Trabzon & 54 & 40.8938 & 39.7113 & Suburban & L & 2016 & 34 & - & - \\
\hline \multirow[t]{3}{*}{ Yalova } & 55 & 40.6217 & 29.1770 & Rural & L & 2016 & 1 & 44 & - \\
\hline & 56 & 40.6085 & 29.2080 & Suburban & L & 2016 & - & 42 & - \\
\hline & 57 & 40.6428 & 29.0968 & Suburban & $L$ & 2016 & - & 40 & - \\
\hline \multirow[t]{3}{*}{ Zonguldak } & 58 & 41.4105 & 32.0890 & Suburban & L & 2016 & 10 & - & - \\
\hline & 59 & 41.3537 & 32.0900 & Suburban & L & 2016 & 15 & - & - \\
\hline & 60 & 41.4529 & 31.8203 & Urban & L & 2016 & 7 & - & - \\
\hline
\end{tabular}

Abbreviations: $\mathrm{A}$, adult; $\mathrm{L}$, larva; na, not applicable

Note: Columns wPip-I, wPip -II and wPip-IV indicate the amount of individuals infected with wPip-I, wPip -II and wPip-IV, respectively, in a given sampling site ${ }^{a}$ Samples from this site were used to establish Tek wPip-I and Tek wPip-II lines

humidity and a 12:12 $\mathrm{h}$ photoperiod) and fed with a mixture of shrimp powder and rabbit pellets. To establish isofemale lines, females were fed with turkey blood using a Hemotek membrane feeding system (Discovery Workshops, Blackburn, United Kingdom) and were allowed to lay eggs five days later. Each egg raft (100-300 eggs) was isolated for hatching, and the isofemale line was established using resulting sibling larvae. A pool of first-instar larvae (L1) was tested to identify the $w$ Pip group. Two isofemale lines each harbouring either a $w$ Pip-I or $w$ Pip-II strain were

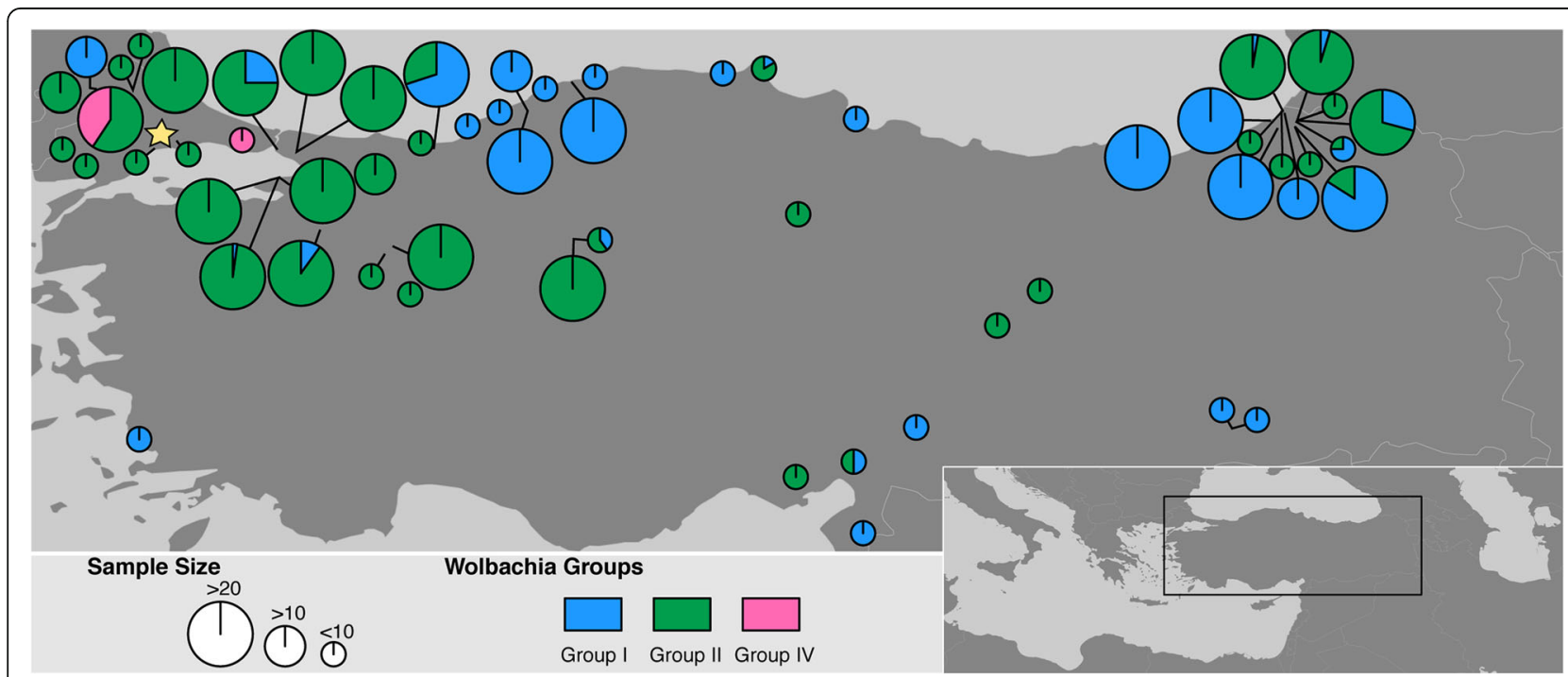

Fig. 1 Sampling sites and diversity of wPip in CX. pipiens (s.l.) populations in Turkey. A total of 753 samples were collected from 59 different sampling sites, tested with a PCR/RFLP assay on the pk1 gene (1.3 kb) and assigned to one of the five genetically distinct wPip groups (wPip-I to V). Results showed the occurrence of wPip from three different groups in the area namely wPip-III and IV. Size of the circle represents the sampling size. Percentage of a given wPip group in a given population is shown in different colours; blue: wPip-l, green: wPip-Il, pink: WPip-IV as defined in Dumas et al. [16]. Star indicates the location of the samples (Tekirdag, sampling site 52) that have been used to establish Tek wPip-l and Tek wPip-ll lines. Reciprocal crosses between these lines and additional reference lines were performed to identify natural $\mathrm{Cl}$ patterns caused by wPip groups in this region 
reared for further crossing experiments in $65 \mathrm{dm}^{3}$ cages in insectary conditions and were fed with a honey solution and a weekly blood meal.

\section{Crossing experiments between Turkish Culex pipiens lines} Tek $w$ Pip-I and Tek $w$ Pip-II isofemale mosquito lines were reared for at least four generations in insectary conditions to allow their acclimatisation before crossing experiments. Mosquitoes were isolated during pupal stage, and emerging adults were sexed. Then, $2-5$ days old virgin males and females $(n=25-50)$ were used to carry out reciprocal crosses between them and with Istanbul $w$ Pip-IV line. Females were fed with turkey blood using a Hemotek membrane feeding system (Discovery Workshops, United Kingdom) on the sixth day following caging and were allowed to lay eggs five days after the blood meal. Egg rafts were then isolated individually in 24 well plates filled with tap water until hatching. Embryonic development of all the unhatched egg rafts was verified to differentiate between nonfertilized egg rafts and CI induced embryonic death as previously described [37].

The crossing relationships were identified as following [7]: compatible (C) when $>90 \%$ of the rafts hatched in the two reciprocal crosses; and incompatible (IC), with two CI patterns: (i) unidirectionally incompatible crosses: when between $0-10 \%$ of the rafts hatched in one of the reciprocal crosses and > 90\% in the other; and (ii) bidirectionally incompatible crosses: when less than $10 \%$ of the rafts hatched in both reciprocal crosses.

\section{Crossing experiments to infer mod and resc functions}

The mod (male crossing type) and resc (female crossing type) functions caused by many $w$ Pip strains, which belong to different $w$ Pip groups $(\mathrm{I}-\mathrm{V})$, have been identified by reciprocal crosses with 4 reference lines: LaVar ( $w$ Pip-II), MaClo, Slab ( $w$ Pip-III) and Istanbul ( $w$ Pip-IV) [7]. Here, we used same four reference lines to define the mod-resc functions of Tek $w$ Pip-I and Tek $w$ Pip-II isofemale lines and to compare them to previously defined ones [7].

\section{Identification of Wolbachia diversity}

DNA was extracted from the samples using CTAB method [38]. PCR assays were conducted using pk1 primers (PK1 Forward: 5'-CCA CTA CAT TGC GCT ATA GA-3' and PK1 Reverse: 5'-ACA GTA GAA CTA CAC TCC TCC A3'-AM397079 [12]), which amplify a 1.3-kilobase (kb) fragment from ankyrin domain coding gene of Wolbachia. PCR amplifications were made in following conditions: initial denaturation for $5 \mathrm{~min}$ at $94{ }^{\circ} \mathrm{C}$, followed by $35 \mathrm{cy}$ cles of denaturation, annealing and elongation respectively at $94{ }^{\circ} \mathrm{C}$ for $30 \mathrm{~s}, 52{ }^{\circ} \mathrm{C}$ for $30 \mathrm{~s}$, and $72{ }^{\circ} \mathrm{C}$ for $90 \mathrm{~s}$, and a final elongation at $72{ }^{\circ} \mathrm{C}$ for $5 \mathrm{mn}$. Resulting PCR products then used in RFLP assays first with TaqaI enzyme to discriminate specific $w$ Pip alleles "a" or "e" ( $w$ Pip- I or $w$ Pip-V; 991, 251, 107 bp), "b” (wPip-III; 669, 665 bp), “c” ( $w$ Pip-II; 851, 498 bp) and "d" (wPip-IV; 497, 251, 107 bp) $[7,16]$. Secondly, since TaqaI digestion of "a" and "e" alleles show the same digestion pattern, pk1 PCR products of the samples showing this pattern were digested with PstI enzyme to further discriminate "a" ( $w$ Pip- I; 903, 303, 141 bp) and "e" ( $w$ Pip-V; 903, $430 \mathrm{bp)}$ ) alleles [7, 16]. Digested amplified fragments were separated by agarose gel electrophoresis $(2 \%)$, stained with ethidium bromide $(1 \mu \mathrm{g} / \mathrm{ml})$ and visualized with UV light. Samples from laboratory mosquito lines with different $w$ Pip groups and tetracyclinecured Wolbachia negative lines were included in every reaction as positive and negative controls, respectively, and always gave the expected result.

\section{Statistical analyses}

The occurrence of different $w$ Pip groups was compared by a Chi-square test using $\mathrm{R}$ software (version 3.3.1).

\section{Results}

Diversity and distribution of wPip groups

Wolbachia wPip was present in all of the $753 C x$. pipiens (s.l.) individuals tested and they were further identifiable to one of the five previously described groups ( $w$ Pip-I to V). Co-infection of one individual by different $w$ Pip groups was never observed. Out of five $w$ Pip groups identified so far in the world, three of them (i.e. $w$ Pip-I-II and IV) were represented in the studied area (Fig. 1). The abundance of these groups was significantly different from each other $\left(\chi^{2}=474.99, d f=2, P<0.0001\right)$. While $w$ Pip-II was the most dominant ( $n=500,66 \%$ of the samples, Table 1 , Fig. 1) and widespread group (39 sampling sites out of 59 total) in Turkey; $w$ Pip-IV was found only in two locations, both in Thrace Region (in Edirne, sampling site 21 and Istanbul sampling site 34, Table 1; Fig. 1), and was the least abundant group $(n=12,1 \%$ of the samples, Table 1; Fig. 1). wPip-I was found in 31 locations and a total of 241 individuals.

\section{Co-existence of wPip strains in different individuals from the same sampling sites}

In $20 \%$ of the sampling sites $w$ Pip-I and $w$ Pip-II co-existed (Table 1, Fig. 1). wPip-IV was only found co-existing with $w$ Pip-II in one sampling site but never found in the same sampling site with $w$ Pip-I, even though they were sampled from nearby sites $(\sim 8 \mathrm{~km})$ in north western Turkey (in Edirne, sampling site 21 and 23, Table 1; Fig. 1).

\section{Naturally occurring $\mathrm{Cl}$ patterns in Turkey}

Two isofemale lines (Tek $w$ Pip-I and Tek $w$ Pip-II), harbouring two different $w$ Pip strains from two different groups, were established from north western Turkey (Tekirdag Province, Table 1 sampling site 52, Fig. 1) to identify CI 
patterns caused by different $w$ Pip groups in the region. Reciprocal crosses between these lines showed that Tek $w$ Pip-I and Tek $w$ Pip-II were fully compatible with each other (Table 2). Both lines were bidirectionally incompatible with the line harbouring Istanbul strain (wPip-IV, Table 2).

\section{Mod and resc properties of Turkish wPip strains}

To compare mod and resc functions of Turkish $w$ Pip strains with mod and resc functions of worldwide collected $w$ Pip strains, we performed reciprocal crosses of Tek $w$ Pip-I and Tek $w$ Pip-II lines with the four reference lines [LaVar (wPip-II), MaClo (wPip-III), Slab (wPip-III) and Istanbul ( $w$ Pip-IV)]. Tek $w$ Pip-I males were compatible with LaVar ( $w$ Pip-II) and MaClo (wPip-III) females while incompatible with Slab (wPip-III) and Istanbul (wPip-IV) females (Table 2). This type of mod property, inferred from similar crosses, has already been shown for the $w$ Pip-I group from Tunisia; numbered "vi" [7]. Contrarily, Tek $w$ Pip-II males demonstrated a new mod property, as they were incompatible with LaVar and Istanbul, and compatible with MaClo and Slab females (Table 2). We numbered this new mod as "ix" to continue the previously published numeration [7]. Both Tek wPip-I and Tek wPip-II lines showed the same resc type, which was characterised by the compatible crosses of females of these lines with all the males from the reference lines except Istanbul (Table 2). This resc type (resc "2") is the most common resc type found worldwide for $w$ Pip-I and $w$ Pip-II groups [7].

\section{Discussion}

In Turkey, all tested Cx. pipiens were infected with Wolbachia $w$ Pip. Such fixation of $w$ Pip has been demonstrated worldwide, including in the neighbouring country Iran [39], in Cx. pipiens and Cx. quinquefasciatus populations [35, 40-44]. A previous study in Turkey, however, showed a lower prevalence of $w$ Pip [45]. This might be caused by the misidentification of a recently described cryptic species within $C x$. pipiens complex that has been shown to lack $w$ Pip infection and to be reproductively isolated from the other members of the complex $[46,47]$. Similarly, $C x$. torrentium, which is difficult to differentiate morphologically from $C x$. pipiens (s.l.) mosquitoes is not infected with Wolbachia [41, 44, 48]. Therefore 100\% $w$ Pip infection rate of our samples confirmed that we only analysed $C x$. pipiens complex members (excluding both previously mentioned cryptic species and $C x$. torrentium) in the present study.

The identification of the Cx. pipiens taxa was left out of the scope of this study for several reasons. Previous studies on the diversification of $w$ Pip in Cx. pipiens (s.l.) have proved that their diversity is not directly related to the nuclear genetic background of the mosquitoes, meaning that no $w$ Pip group was specific for a $C x$. pipiens sibling species $[10,21,49]$. It rather follows the same distribution as mitochondrial diversity (mtDNA) of mosquitoes, as $w$ Pip are maternally transmitted to the next generation through the egg cytoplasm along with mitochondria [16, 46]. Moreover, CI properties are independent of the genetic background of Cx. pipiens (s.l.) and directly dictated by their Wolbachia [20]. Recent studies on Cx. pipiens (s.l.) in Turkey had shown that both $C x$. quinquefasciatus, $C x$. pipiens and its form $C x$. pipiens $\mathrm{f}$. molestus, were present in Turkey [24]. The co-existence of these sibling species in same sampling sites $[24,50]$ and the existence of hybrids [50-52] suggest that they can exchange $w$ Pip strains easily in natural populations.

We have identified three different $w$ Pip groups, i.e. $w$ Pip-I-II and IV in Cx. pipiens (s.l.) mosquito populations in Turkey. The only previous sample from Turkey, which has been assigned to $w$ Pip groups, was a $w$ Pip-IV group strain collected in Istanbul in 2003 [16, 35]. Other than this single case, the $w$ Pip diversity in Turkish $C x$.

Table 2 Crossing relationships between lines from Turkey (Tek wPip-l \& Tek wPip-II) and reference laboratory Wolbachia strains

\begin{tabular}{|c|c|c|c|c|c|c|c|}
\hline & Males & $\begin{array}{l}\text { Tek } \\
\text { wPip-I }\end{array}$ & $\begin{array}{l}\text { Tek } \\
\text { wPip-II }\end{array}$ & $\begin{array}{l}\text { Istanbul } \\
\text { wPip-IV }\end{array}$ & $\begin{array}{l}\text { Slab } \\
\text { wPip-III }\end{array}$ & $\begin{array}{l}\text { LaVar } \\
\text { wPip-\|l }\end{array}$ & $\begin{array}{l}\text { MaClo } \\
\text { wPip-III }\end{array}$ \\
\hline & Mod & vi & ix & viii & & & \\
\hline Females & Resc & & & & & & \\
\hline Tek I & 2 & & C (24) & IC (24) & C (9) & C (17) & C (34) \\
\hline Tek II & 2 & C (26) & & IC (32) & C (26) & C (20) & C (14) \\
\hline Istanbul & 3 & IC (58) & IC (36) & & IC (34) & IC $(40)^{a}$ & $C(31)^{a}$ \\
\hline Slab & & IC (32) & C (27) & $C(33)^{a}$ & & IC $(30)^{\mathrm{a}}$ & IC (99) \\
\hline LaVar & & C (15) & IC (33) & IC $(26)^{\mathrm{a}}$ & $C(8)^{a}$ & & $C(10)^{a}$ \\
\hline MaClo & & C (18) & C (20) & IC $(53)^{a}$ & $C(43)^{a}$ & $C(36)^{\mathrm{a}}$ & \\
\hline
\end{tabular}

${ }^{\mathrm{a}}$ Data taken from Duron et al. [10]

Note: Reciprocal crosses between Tek wPip-I, Tek wPip-II, Istanbul wPip-IV lines have been performed to identify natural Cl patterns induced by these strains in the region. Additional reciprocal crosses between Turkish lines and 4 reference laboratory lines [LaVar (wPip-II), MaClo and Slab (wPip-III) and Istanbul (wPip-IV)] have been performed to define the mod-resc functions of Tek wPip-I and Tek WPip-II isofemale lines and to compare them to previously defined ones by Atyame et al. [7]. Crosses were classified as either compatible (C, raft hatching $>90 \%$ ) or incompatible (IC, raft hatching $=0-10 \%)$. Bidirectionally incompatible crosses are shown in bold. The number of egg-rafts collected for each cross is indicated in parentheses 
pipiens populations was to date completely unknown. Although $w$ Pip diversity was investigated in regions around Turkey [16]. Dumas et al. [16] have found wPipI strains in Middle East (Lebanon, Israel, Jordan) and in northern Africa (Tunisia), wPip-II strains widely distributed in eastern Europe and Cyprus, and $w$ Pip-IV strains in a patchy distribution in Europe -in areas dominated by other $w$ Pip groups. We demonstrated that $w$ Pip strains belonging to $w$ Pip-I, $w$ Pip-II and $w$ Pip-IV, previously identified near Turkey, are all present in the Cx. pipiens (s.l.) populations within this country, suggesting that Turkey is a crossroads for $w$ Pip strains from eastern Europe, Africa and Middle East as for their vector hosts.

The most widespread groups in Turkey, $w$ Pip-I and $w$ Pip-II, induce reciprocal compatibility between their hosts and co-exist in many populations. Indeed, different $w$ Pip strains can co-exist in a single natural $C x$. pipiens (s.l.) population [12, 35, 43, 53] and these coexisting strains are usually compatible with each other [43]. Mathematical models confirm that only compatible strains can stably coexist in unstructured and panmictic host populations when the fitness costs related to infection by different $w$ Pip strains are the same $[54,55]$. When bidirectional incompatibility inducing $w$ Pip strains co-exist in one population the most prevalent strain is expected to eventually invade the population $[54,55]$ and when unidirectional incompatibility inducing $w$ Pip strains co-exist, CI-inducing strain is expected to invade the population once above a frequency threshold $[49,55]$. Therefore, a stable co-existence of incompatible strains is predicted to be rare. However, an example of the co-existence of unidirectional incompatibility inducing strains, belonging to a $w$ Pip-I group and $w$ Pip-IV group, has been shown in Tunisia while mathematical models predicted that $w$ Pip-I should have invaded this area in only 4 generations [5, 49]. Atyame et al. [49] hypothesized that low dispersal and extinction-recolonization events could explain this stable co-existence. In one site in Turkey, we observed the co-existence of bidirectional incompatibility inducing strains ( $w$ Pip-IV and $w$ Pip-II). The low prevalence of $w$ Pip-IV and its incompatibilities suggest that it should disappear from the population. However, we have evidence that $w$ Pip-IV strains were already present at least 13 years ago at Istanbul since it has been sampled in 2003 [35]. This persistence of $w$ Pip-IV, at low frequencies, could be explained by higher fitness costs associated with $w$ Pip-I and $w$ Pip-II infections or by extinction-recolonization events of $w$ Pip-IV-infected individuals as it has been suspected in Tunisia $[43,49,55]$. Fitness difference could be, for instance, linked to differences in fecundity $[56,57]$ or to a possible ability of the different $w$ Pip strains to protect their hosts against other microbial infections [58-62]. Further studies on the differences between $w$ Pip strains in terms of infection costs and pathogen protection might help to understand stable co-existence of bidirectionally incompatible $w$ Pip-IV strains observed in Turkey.

To study the phenotypical diversity of crossing types in Turkey, we crossed Turkish isofemale lines harbouring $w$ Pip-I and $w$ Pip-II strains with four reference lines defined by Atyame et al. [7]. We inferred both their mod and resc functions and compared them to the eight mod and four resc functions already described worldwide. The Tek $w$ Pip-I line showed the most common resc functions for a $w$ Pip-I infected line (i.e. resc 2) but a rare mod function previously defined in few lines harbouring $w$ Pip-I or $w$ Pip-II strains (i.e. $\bmod$ "vi"). The Tek $w$ Pip-II line showed the same resc function as $w$ Pip-I (i.e. resc 2 ) but a totally new mod function (i.e. $\bmod$ "ix"). Our findings are consistent with theoretical predictions and empirical data suggesting new mod functions can more easily evolve and spread in the population than new resc functions $[7,63]$.

Natural CI properties induced by Wolbachia can be used to control the vector populations: the mass release of males harbouring incompatible Wolbachia into the natural populations can decrease the female reproduction and eradicate the pest/ vector populations (IIT) [29, 30]. Indeed, $w$ Pip induced $C I$ has been used against $C x$. quinquefasciatus (formerly named Cx. pipiens fatigans) for the first time in 1967 to control filariasis in Southeast Asia [31]. More recently, natural CI properties caused by $w$ Pip infection have been found promising to control $C x$. pallens (no longer considered as a valid species) in China [32] and $C x$. pipiens populations in La Réunion Island [33, 34]. In the latter study, a $w$ Pip-IV strain from Istanbul has been successfully used to sterilise $w$ Pip-I females in semifield conditions. We demonstrated that this Istanbul strain also induces bidirectional incompatibility with mosquitoes harbouring $w$ Pip-I or $w$ Pip-II in Turkey. This means that most Cx. pipiens females in Turkey, except in few sites in the Thrace region, can be sterilised by the release of males infected with Istanbul strain. Although further studies on intrapopulation $\mathrm{CI}$ variability, mating choice, hatching rate and population dynamics in semi field populations are needed for $w$ Pip-IV Istanbul to be used in future vector control programs in Turkey, a critical region for vector-borne diseases, our results suggest that it could constitute a good candidate.

\section{Conclusions}

We identified $w$ Pip diversity in natural $C x$. pipiens (s.l.) populations in Turkey. The previously described $w$ PipIV group was in fact restricted to only two populations while $w$ Pip-I and $w$ Pip-II group are widely distributed and coexist in many populations all over the country. The $w$ Pip-IV strain Istanbul was found bidirectionally incompatible with individuals harbouring $w$ Pip-I or $w$ PipII from Turkey. This highlights the potential of $w$ Pip-IV 
harbouring males as a vector control to sterilise local $C x$. pipiens populations, particularly where only $w$ Pip-I or $w$ Pip-II harbouring females were found.

\section{Abbreviations}

C: Compatible; Cl: Cytoplasmic incompatibility; IC: Incompatible; Mod: Modification ability; Resc: Rescue ability; wPip: Wolbachia pipientis

\section{Acknowledgements}

We are thankful to Dr Hilal Bedir (Kafkas University, Kars, Turkey), Dr Berna Demirci (Kafkas University, Kars, Turkey) and Dr Mustafa Akiner (RTE University, Rize, Turkey) for their guidance during fieldwork, to Dr Sırrı Kar (Namık Kemal University, Tekirdağ, Turkey) for the collection of Cx. pipiens from Tekirdağ (Turkey), to Patrick Makoundou, Sandra Unal and Marco Perriat-Sanguinet from team EVAS (ISEM) for technical support.

\section{Funding}

This work was funded by the French ANR program (project ANR-16-CE02-0006-01 "CIAWOL", 2017-2020). Mine Altinli received support from Ministère des affaires étrangères (Campus France) via the French Embassy in Turkey and Project "ISEM Sud", 2016 for her PhD.

\section{Availability of data and materials}

All data generated or analysed during this study are included in this published article.

\section{Authors' contributions}

MA, FG, MW and MS contributed to the conception of the study. MA, BA, FG did the sample collection. MA and FG identified samples, MA did the Wolbachia genotyping. MA and MS carried out crossing experiments. MA analysed the data and interpreted the results. MA and MS drafted the article. All authors read and approved the final manuscript.

\section{Ethics approval and consent to participate}

Not applicable.

\section{Consent for publication}

Not applicable.

\section{Competing interests}

The authors declare that they have no competing interests.

\section{Publisher's Note}

Springer Nature remains neutral with regard to jurisdictional claims in published maps and institutional affiliations.

\section{Author details}

${ }^{1}$ Institut des Sciences de l'Evolution de Montpellier (CNRS-Université de Montpellier-IRD-EPHE), Montpellier, France. ${ }^{2}$ Faculty of Sciences, Department of Biology, Division of Ecology, VERG Laboratories, Hacettepe University, Ankara, Turkey.

Received: 11 January 2018 Accepted: 6 March 2018

\section{Published online: 20 March 2018}

\section{References}

1. Hertig M. The Rickettsia, Wolbachia pipientis (gen. et sp. n.) and associated inclusions of the mosquito, Culex pipiens. Parasitology. 1936;28:453-86.

2. Weinert LA, Araujo-Jnr EV, Ahmed MZ, Welch JJ. The incidence of bacterial endosymbionts in terrestrial arthropods. P Roy Soc B Biol Sci. 2015;282:20150249.

3. Werren JH, Baldo L, Clark ME. Wolbachia: master manipulators of invertebrate biology. Nat Rev Microbiol. 2008;6:741-51.

4. Werren JH. Biology of Wolbachia. Annu Rev Entomol. 1997;124:587-609.

5. Hoffmann a. a., Turelli M, Harshman LG. Factors affecting the distribution of cytoplasmic incompatibility in Drosophila simulans. Genetics. 1990;126:933-48.

6. Yen JH, Barr AR. New hypothesis of the cause of cytoplasmic incompatibility in Culex pipiens L. Nature. 1971;232:657-8.

7. Atyame CM, Labbé $P$, Dumas E, Milesi $P$, Charlat $S$, Fort $P$, et al. Wolbachia divergence and the evolution of cytoplasmic incompatibility in Culex pipiens. PLoS One. 2014;9:e87336.
8. Farajollahi A, Fonseca DM, Kramer LD, Marm Kilpatrick A. "Bird biting" mosquitoes and human disease: a review of the role of Culex pipiens complex mosquitoes in epidemiology. Infect Genet Evol. 2011;11:1577-85.

9. Laven H. Speciation and evolution in Culex pipiens. In: Wright JW, Pal R, editors. Genetics of Insect Vectors of Disease. Amsterdam: Elsevier; 1967. p. 251-75.

10. Duron O, Bernard C, Unal S, Berthomieu A, Berticat C, Weill M. Tracking factors modulating cytoplasmic incompatibilities in the mosquito Culex pipiens. Mol Ecol. 2006;15:3061-71.

11. Wolfgang A, Markus R, Dimitrios NA, Christian S. Evidence for low-titre infections in insect symbiosis: Wolbachia in the bark beetle Pityogenes chalcographus (Coleoptera, Scolytinae). Environ Microbiol. 2009;11:1923-33.

12. Atyame CM, Delsuc F, Pasteur N, Weill M, Duron O. Diversification of Wolbachia endosymbiont in the Culex pipiens mosquito. Mol Biol Evol. 2011;28:2761-72.

13. Guillemaud T, Pasteur N, Rousset F. Contrasting levels of variability between cytoplasmic genomes and incompatibility types in the mosquito Culex pipiens. P Roy Soc B Biol Sci. 1997;264:245-51.

14. Baldo L, Hotopp JCDD, Jolley KA, Bordenstein SR, Biber SA, Choudhury RR, et al. Multilocus sequence typing system for the endosymbiont Wolbachia pipientis. Appl Environ Microbiol. 2006;72:7098-110.

15. Duron O, Boureux A, Echaubard P, Berthomieu A, Berticat C, Fort P, et al. Variability and expression of ankyrin domain genes in Wolbachia variants infecting the mosquito Culex pipiens. J Bacteriol. 2007;189:4442-8.

16. Dumas E, Atyame CM, Milesi P, Fonseca DM, Shaikevich EV, Unal S, et al. Population structure of Wolbachia and cytoplasmic introgression in a complex of mosquito species. BMC Evol Biol. 2013;13:181.

17. Bonneau M, Atyame C, Beji M, Justy F, Cohen-Gonsaud M, Sicard M, et al. Culex pipiens crossing type diversity is governed by an amplified and polymorphic operon in Wolbachia genome. Nat Commun. 2018;9:319.

18. LePage DP, Metcalf JA, Bordenstein SR, On J, Perlmutter JI, Shropshire JD, et al. Prophage WO genes recapitulate and enhance Wolbachia-induced cytoplasmic incompatibility. Nature. 2017;543:243.

19. Beckmann JF, Ronau JA, Hochstrasser M, Tillett D, Ginalski K. A Wolbachia deubiquitylating enzyme induces cytoplasmic incompatibility. Nat Microbiol. 2017;2:17007.

20. Duron O, Bernard J, Atyame CM, Dumas E, Weill M. Rapid evolution of Wolbachia incompatibility types. P Roy Soc B Biol Sci. 2012;279:4473-80.

21. Atyame CM, Duron O, Tortosa P, Pasteur N, Fort P, Weill M. Multiple Wolbachia determinants control the evolution of cytoplasmic incompatibilities in Culex pipiens mosquito populations. Mol Ecol. 2011;20:286-98.

22. Inci A, Yildirim A, Duzlu O, Doganay M, Aksoy S. Tick-borne diseases in Turkey: A review based on one health perspective. PLoS Neglect Trop D. 2016;10:e0005021.

23. Failloux A-B, Bouattour A, Faraj C, Gunay F, Haddad N, Harrat Z, et al. Surveillance of arthropod-borne viruses and their vectors in the Mediterranean and Black Sea regions within the MediLabSecure Network. Curr Trop Med Rep. 2017;4:27-39.

24. Gunay F, Alten B, Simsek F, Aldemir A, Linton Y-M. Barcoding Turkish Culex mosquitoes to facilitate arbovirus vector incrimination studies reveals hidden diversity and new potential vectors. Acta Trop. 2015;143:112-20.

25. Ergunay K, Gunay F, Oter K, Kasap OE, Orsten S, Akkutay AZ, et al. Arboviral surveillance of field-collected mosquitoes reveals circulation of West Nile virus lineage 1 strains in Eastern Thrace, Turkey. Vector-Borne Zoonot. 2013; 13:744-52.

26. Ergunay K, Gunay F, Erisoz Kasap O, Oter K, Gargari S, Karaoglu T, et al. Serological, molecular and entomological surveillance demonstrates widespread circulation of West Nile virus in Turkey. PLoS Neglect Trop D. 2014;8:1-10.

27. Ergunay K, Bakonyi T, Nowotny N, Ozkul A. Close relationship between West Nile virus from Turkey and lineage 1 strain from Central African Republic. Emerg Infect Dis. 2015;21:352-5.

28. Öncü C, Brinkmann A, Günay F, Kar S, Öter K, Sarıaya Y, et al. West Nile virus, Anopheles flavivirus, a novel flavivirus as well as Merida-like rhabdovirus Turkey in field-collected mosquitoes from Thrace and Anatolia. Infect Genet Evol. 2018;57:36-45.

29. Knipling EF, Laven H, Craig GB, Pal R, Kitzmiller JB, Smith CN, et al. Genetic control of insects of public health importance. Bull World Health Organ. 1968:38:421-38.

30. Zabalou S, Riegler M, Theodorakopoulou M, Stauffer C, Savakis C, Bourtzis K. Wolbachia-induced cytoplasmic incompatibility as a means for insect pest population control. Proc Natl Acad Sci USA. 2004;101:15042-5.

31. Laven $\mathrm{H}$. Eradication of Culex pipiens fatigans through cytoplasmic incompatibility. Nature. 1967;216:383-4. 
32. Chen L, Zhu C, Zhang D. Naturally occurring incompatibilities between different Culex pipiens pallens populations as the basis of potential mosquito control measures. PLoS Neglect Trop D. 2013;7:e2030.

33. Atyame CM, Pasteur N, Dumas E, Tortosa P, Tantely ML, Pocquet N, et al. Cytoplasmic incompatibility as a means of controlling Culex pipiens quinquefasciatus mosquito in the islands of the South-Western Indian Ocean. PLoS Neglect Trop D. 2011;5:e1440.

34. Atyame CM, Cattel J, Lebon C, Flores O, Dehecq J-S, Weill M, et al. Wolbachia-based population control strategy targeting Culex quinquefasciatus mosquitoes proves efficient under semi-field conditions. PLoS One. 2015;10:e0119288.

35. Duron O, Lagnel J, Raymond M, Bourtzis K, Fort P, Weill M. Transposable element polymorphism of Wolbachia in the mosquito Culex pipiens: evidence of genetic diversity, superinfection and recombination. Mol Ecol. 2005:14:1561-73.

36. Schaffner E, Angel G, Geoffroy B, Hervy JP, Rhaiem A, Brunhes J. The mosquitoes of Europe. Montpellier. France: Institute de Recherche pour le Développement (IRD); 2001.

37. Duron O, Weill M. Wolbachia infection influences the development of Culex pipiens embryo in incompatible crosses. Heredity. 2006;96:493-500.

38. Rogers SO, Bendich AJ. Extraction of DNA from plant tissues. In: Gelvin SB, Schilperoort RA, Verma DPS, editors. Plant Molecular Biology Manuel. Dordrecht: Springer Netherlands; 1989. p. 73-83.

39. Karami M, Moosa-kazemi SH, Oshaghi MA, Vatandoost H, Sedaghat MM, Rajabnia R. Wolbachia endobacteria in natural populations of Culex pipiens of Iran and its phylogenetic congruence. J Arthropod-Borne Di. 2016;10:349-65.

40. Rasgon JL, Scott TW. Cytoplasmic incompatibility in the California Culex pipiens mosquito species complex: parameter estimates and infection dynamics in natural populations. Genetics. 2003;165:2029-38.

41. Fedorova MV, Shaikevich EV. Morphological and molecular-genetic distinctions between adult mosquitoes Culex torrentium Martini and C. pipiens Linnaeus (Diptera, Culicidae) from Moscow Province. Entomol Rev. 2007:87:127-35.

42. Khrabrova NV, Bukhanskaya ED, Sibataev AK, Volkova TV. The distribution of strains of endosymbiotic bacteria Wolbachia pipientis in natural populations of Culex pipiens mosquitoes (Diptera: Culicidae). Eur Mosq Bull. 2009;27:18-22.

43. Duron O, Raymond M, Weill M. Many compatible Wolbachia strains coexist within natural populations of Culex pipiens mosquito. Heredity. 2011;106:986-93.

44. Raharimalala FN, Boukraa S, Bawin T, Boyer S, Francis F. Molecular detection of six (endo-) symbiotic bacteria in Belgian mosquitoes: first step towards the selection of appropriate paratransgenesis candidates. Parasitol Res. 2016;115:1391-9.

45. Yildirim A, Inci A, Duzlu O, Onder Z, Ciloglu A. Detection and molecular characterization of the Wolbachia endobacteria in the Culex pipiens (Diptera: Culicidae) specimens collected from Kayseri province of Turkey. Ankara Univ Vet Fak. 2013;60:189-94

46. Rasgon $\mathrm{J}$, Cornel AJ, Scott TW. Evolutionary history of a mosquito endosymbiont revealed through mitochondrial hitchhiking. P Roy Soc B Biol Sci. 2006;273:1603-11.

47. Dumas E, Atyame CM, Malcolm CA, Le Goff G, Unal S, Makoundou P, et al. Molecular data reveal a cryptic species within the Culex pipiens mosquito complex. Insect Mol Biol. 2016;25:800-9.

48. Leggewie M, Krumkamp R, Badusche M, Heitmann A, Jansen S, SchmidtChanasit J, et al. Culex torrentium mosquitoes from Germany are negative for Wolbachia. Med Vet Entomol. 2017:1-6.

49. Atyame CM, Labbé P, Rousset F, Beji M, Makoundou P, Duron O, et al. Stable coexistence of incompatible Wolbachia along a narrow contact zone in mosquito field populations. Mol Ecol. 2015;24:508-21.

50. Shaikevich EV, Vinogradova EB, Bouattour A, Gouveia de Almeida AP. Genetic diversity of Culex pipiens mosquitoes in distinct populations from Europe: contribution of $C x$. quinquefasciatus in Mediterranean populations. Parasit Vectors. 2016:9:47.

51. Shaikevich EV, Vinogradova EB. The discovery of a hybrid population of mosquitoes of the Culex pipiens L. complex (Diptera, Culicidae) on the Kos Island (Greece) by means of molecular markers. Entomol Rev. 2014;94:35-9.

52. Smith JL, Fonseca DM. Rapid assays for identification of members of the Culex (Culex) pipiens complex, their hybrids, and other sibling species (Diptera: Culicidae). Am J Trop Med Hyg. 2004;70:339-45.

53. Duron $\mathrm{O}$, Fort $\mathrm{P}$, Weill M. Hypervariable prophage WO sequences describe an unexpected high number of Wolbachia variants in the mosquito Culex pipiens. P Roy Soc B Biol Sci. 2006;273:495-502
54. Rousset F, Raymond M, Kjellberg F. Cytoplasmic incompatibility in the mosquito Culex pipiens: how to explain a cytotype polymorphism? J Evol Biol. 1991:4:69-81.

55. Engelstädter J, Telschow A. Cytoplasmic incompatibility and host population structure. Heredity. 2009;103:196-207.

56. Poinsot D, Merçot H. Wolbachia infection in Drosophila simulans: does the female host bear a physiological cost? Evolution. 1997;51:180-6.

57. Bordenstein SR, Werren JH. Do Wolbachia influence fecundity in Nasonia vitripennis? Heredity. 2000;84:54-62.

58. Hedges LM, Brownlie JC, O'Neill SL, Johnson KN. Wolbachia and virus protection in insects. Science. 2008:322:702.

59. Brownlie JC, Johnson KN. Symbiont-mediated protection in insect hosts. Trends Microbiol. 2009;17:348-54.

60. Hughes GL, Koga R, Xue P, Fukatsu T, Rasgon JL. Wolbachia infections are virulent and inhibit the human malaria parasite Plasmodium falciparum in Anopheles gambiae. PLoS Pathog. 2011;7:3-10.

61. Braquart-Varnier C, Altinli M, Pigeault R, Chevalier FD, Grève P, Bouchon D, et al. The mutualistic side of Wolbachia-isopod interactions: Wolbachia mediated protection against pathogenic intracellular bacteria. Front Microbiol. 2015;6:1388.

62. Teixeira L, Ferreira Á, Ashburner M. The bacterial symbiont Wolbachia induces resistance to RNA viral infections in Drosophila melanogaster. PLoS Biol. 2008;6:2753-63.

63. Charlat S, Calme C, Merçot H. On the mod resc model and the evolution of Wolbachia compatibility types. Genetics. 2001;159:1415-22.

\section{Submit your next manuscript to BioMed Central and we will help you at every step:}

- We accept pre-submission inquiries

- Our selector tool helps you to find the most relevant journal

- We provide round the clock customer support

- Convenient online submission

- Thorough peer review

- Inclusion in PubMed and all major indexing services

- Maximum visibility for your research

Submit your manuscript at www.biomedcentral.com/submit

) Biomed Central 